\title{
Quality of Resuscitative Care Provided to an Infant with Abusive Head Trauma in
}

\section{Community Emergency Departments: An In Situ, Prospective Simulation-Based Study}

\author{
Authors: \\ Riad Lutfi, MD 1 \\ Department of Pediatrics, Division of Pediatric Critical Care \\ Indiana University School of Medicine and Riley Hospital for Children at Indiana \\ University Health \\ 705 Riley Hospital Drive, Riley Phase 2 Room 4900 \\ Indianapolis, Indiana 46202-5225 \\ Email: rlutfi@iu.edu \\ Zachary J Berrens, MD ${ }^{1}$ \\ Department of Pediatrics, Division of Pediatric Critical Care \\ Indiana University School of Medicine and Riley Hospital for Children at Indiana \\ University Health \\ 705 Riley Hospital Drive, Riley Phase 2 Room 4900 \\ Indianapolis, Indiana 46202-5225 \\ Email: zberrens@iu.edu \\ Laurie L Ackerman, MD² \\ Department of Neurosurgery, \\ Indiana University School of Medicine and Riley Hospital for Children at Indiana \\ University Health \\ 705 Riley Hospital Drive, Room 1601 \\ Indianapolis, Indiana 46202-5225 \\ Email: lackerma@iuhealth.org \\ Erin E Montgomery, $\mathrm{RN}^{3}$ \\ LifeLine Critical Care Transport, Indiana University Health \\ Indianapolis, Indiana 46202-5225 \\ Email:white20@iu.edu \\ Manahil Mustafa, MBBS 1 \\ Department of Pediatrics, Division of Pediatric Critical Care \\ Indiana University School of Medicine and Riley Hospital for Children at Indiana \\ University Health \\ 705 Riley Hospital Drive, Riley Phase 2 Room 4900 \\ Indianapolis, Indiana 46202-5225 \\ Email: mmmustaf@iu.edu \\ Michele L Kirby, $\mathrm{RN}^{3}$ \\ LifeLine Critical Care Transport, Indiana University Health \\ Indianapolis, Indiana 46202-5225 \\ Email: mlbaumga@iu.edu \\ Kellie J. Pearson, RRT ${ }^{3}$
}

This is the author's manuscript of the article published in final edited form as:

Lutfi, R., Berrens, Z. J., Ackerman, L. L., Montgomery, E. E., Mustafa, M., Kirby, M. L., .. \& Abulebda, K. (2022). Quality of resuscitative care provided to an infant with abusive head trauma in community emergency departments: an in situ, prospective, simulation-based study. Pediatric Emergency Care, 38(1), e337-e342. https://doi.org/10.1097/PEC.0000000000002277 
LifeLine Critical Care Transport, Indiana University Health

Indianapolis, Indiana 46202-5225

Email: kleeper@iuhealth.org

Samer Abu-Sultaneh, MD ${ }^{1}$

Department of Pediatrics, Division of Pediatric Critical Care

Indiana University School of Medicine and Riley Hospital for Children at Indiana

University Health

705 Riley Hospital Drive, Riley Phase 2 Room 4900

Indianapolis, Indiana 46202-5225

Email: sultaneh@iu.edu

Kamal Abulebda, MD ${ }^{1}$

Department of Pediatrics, Division of Pediatric Critical Care

Indiana University School of Medicine and Riley Hospital for Children at Indiana

University Health

705 Riley Hospital Drive, Riley Phase 2 Room 4900

Indianapolis, Indiana 46202-5225

Email: kabulebd@iu.edu

${ }^{1}$ Division of Pediatric Critical Care Medicine, Department of Pediatrics, Indiana University School of Medicine and Riley Hospital for Children at Indiana University Health, Indianapolis, Indiana

${ }^{2}$ Department of Neurosurgery, Indiana University School of Medicine and Riley Hospital for Children at Indiana University Health, Indianapolis, Indiana

${ }^{3}$ LifeLine Critical Care Transport, Indiana University Health, Indianapolis, Indiana

\section{Corresponding Author:}

Riad Lutfi, MD, FAAP

Department of Pediatrics, Division of Pediatric Critical Care

Indiana University School of Medicine and Riley Hospital for Children at Indiana

University Health

705 Riley Hospital Drive, Riley Phase 2 Room 4900

Indianapolis, Indiana 46202-5225

Phone: 317- 944-1405

Fax: 317-944-7267

Email: rlutfi@iu.edu

\section{Declarations}

\section{Ethics approval and consent to participate}

This study was administratively reviewed by the Institutional Review Board of Indiana University and determined to be not human subjects research.

Number 1509259968 
Not Human Subjects

\section{Consent for publication}

Not applicable

\section{Availability of data and material}

The datasets used and/or analyzed during the current study are available from the corresponding author on reasonable request.

\section{Competing interests}

The authors declare that they have no competing interests

\section{Funding}

Indiana university health values fund for education: VFE-332-Lufti

\section{Authors' contributions}

Drs. RL, SA and KA were the leaders of this project and were involved in the conception and design of the project, reviewed the available pertinent literature, data collection, drafted of the initial manuscript, and gave final approval of the manuscript.

All authors were involved in the conception and design of the project, met regularly with the project leader to review preliminary data, performed critical revisions of the manuscript, and granted final approval of the manuscript.

\section{Acknowledgements}

We would like to thank the members of pediatric critical care division at Riley Hospital for Children at IU health and Indiana University Health Lifeline for their support and help in this project. 


\section{Quality of Resuscitative Care Provided to an Infant with Abusive Head Trauma in Community Emergency Departments: An In Situ, Prospective Simulation-Based Study}

Abstract

Objectives: Abusive head trauma (AHT) is a very common and serious form of physical abuse, and a major cause of mortality and morbidity for young children. Early Recognition and supportive care of children with AHT is a common challenge in community emergency department (CEDs). We hypothesized that standardized, in situ simulation can be used to measure and compare the quality of resuscitative measures provided to children with AHT in a diverse set of CEDs.

Methods: This prospective, simulation-based study measured teams' performance across CEDs. The primary outcome was overall adherence to AHT using a 15 -item performance assessment checklist based on the number of tasks performed correctly on the checklist.

Results: Fifty three multi-professional teams from 18 CEDs participated in the study. Out of 270 participants, $20.7 \%$ were physicians, $65.2 \%$ registered nurses, and $14.1 \%$ were other providers. Out of all tasks, assessment of airway/breathing was the most successfully conducted task by $53 / 53$ teams (100\%). Although $43 / 53$ teams ( $81 \%$ ) verbalized the suspicion for AHT, only 21/53 teams (39.6\%) used hyperosmolar agent, 4/53 teams ( $7.5 \%)$ applied cervical spine collar stabilization, and 6/53 teams (11.3\%) raised the head of the bed. No significant difference in adherence to the checklist was found in the CEDs with an inpatient pediatric service or these with designated adult 
trauma centers compared to CEDs without. CEDs closer to the main academic center outperformed CEDs these that are further away.

Conclusion: This study used in situ simulation to describe quality of resuscitative care provided to an infant presenting with AHT across a diverse set of CEDs, revealing variability in the initial recognition and stabilizing efforts and provided and targets for improvement. Future interventions focusing on reducing these gaps could improve the performance of CED providers and lead to improved patient outcomes.

\section{Introduction}

Child maltreatment is a considerable social and public health problem in the United States. Reports from the Department of Human and Health Services showed that 674,000 (9.1 victims per 1,000 children in the population) were identified as victims of child abuse and neglect with a nationally estimated 1,720 children died from abuse or neglect in $2017^{1}$. This equates to a rate of 2.32 children per 100,000 children in the general population or an average of nearly five children dying every day from abuse or neglect ${ }^{1}$.

Physical abuse represents $18.3 \%$ of all children maltreatment ${ }^{2-4}$. Abusive head trauma (AHT) in particular is a very common and the most serious form of physical abuse. AHT is a major cause of severe traumatic brain injury (TBI) in young children. It accounts for $80 \%$ of death resulting from child maltreatment injuries, making it the leading cause of 
traumatic death for children younger than 1 year of age ${ }^{3,5,6}$. Additionally, two-thirds of children who survive AHT have significant neurologic consequences.

AHT is difficult to diagnose and is often missed due to misleading history, subtle nonspecific presentation, and lack of consistent signs of injury ${ }^{7}$. Children with AHT initially present at a diverse set of emergency departments where frontline providers must recognize and provide immediate treatment ${ }^{8,9}$. The majority of these children $(>90 \%)$ seek care in community emergency departments (CEDs) that are not solely prepared to care for children ${ }^{10}$. Several studies show that physical abuse is more commonly missed at CEDs, and that fewer children are diagnosed with an AHT at CEDs in comparison to pediatric EDs due to misdiagnosis and other challenges innate to working in CEDs ${ }^{9,11}$. This is aligned with national reports showing CEDs are generally less prepared than pediatric centers and have limited access to resources, guidelines, and pediatric services and specialists due to a limited national specialist workforce ${ }^{12,13,10,14}$. Although data reveals disparities in the quality of care and outcomes of pediatric trauma patients presenting to CEDs in comparison to pediatric EDs, there is limited data describing the quality of care and management provided to patients with AHT presenting to CEDs. Research in pediatric AHT has mainly focused on deficiencies in CEDs recognition of AHT and reluctance of providers to report suspicious injuries. Given the significant morbidity and high fatality in pediatric patients with AHT compared to non-abusive brain injury, it is important to accurately describe current practices among CEDs providers regarding acute supportive care of AHT and to identify opportunities for improvement ${ }^{15}$. 
Research describing the recognition of AHT among children in CEDs is limited by an underestimated incidence of AHTs, cost and logistic difficulties. Additionally, the lack of clear consensus regarding goals of management and treatment of AHT contributes to the lack of standardized guidelines once AHT is diagnosed in the CED ${ }^{16}$. Therefore, we leveraged the use of in situ simulation as an investigative methodology to measure the quality of care to children with AHT in CED settings. In situ simulation provides a robust tool for the measurement of the processes of patient care and adherence to standards of care ${ }^{17,18}$. In situ simulation allows the research team to evaluate the quality of care delivered by multi-disciplinary healthcare teams using their own equipment and clinical resources ${ }^{19}$. Simulation has developed into a valuable tool to measure clinical performance and discover safety threats and system issues in high-risk clinical settings $20,21$.

The objectives of this study were to 1) evaluate the quality of resuscitative care in CEDs caring for an infant presenting with a severe AHT in simulated setting and to 2) assess changes in providers self-reported comfort in managing pediatric patients with AHT presenting to their emergency department after pediatric AHT simulation.

\section{Materials and Methods:}

$\underline{\text { Study Design, Setting and Population: }}$

This is a prospective simulation-based study designed to measure the performance of multi-professional teams providing stabilization efforts in a simulated 3-month old infant 
presenting with severe AHT. The Institutional Review Board at Indiana University School of Medicine approved this study.

The study team research coordinator contacted sites through established relationships at each CED. All site visits were scheduled in coordination with each hospital's CED medical director and/or manager. The study was conducted between January 2018 and February 2019 across 18 CEDs with varying patient volumes and locations throughout the state of Indiana to provide a diverse representation of CEDs. Staff was recruited to participate in the simulation sessions by the site manager or medical director who served as a pediatric champion for their site. The CED participant teams were composed of registered nurses $(\mathrm{RN})$, respiratory therapists $(\mathrm{RT})$, emergency medicine physicians, and physician assistants. Pharmacists and emergency medical services were also included if available, since they are normally part of the resuscitation team. Team size was limited to 4 to 6 participants per simulation scenario to reflect the actual clinical setting. All participants were protected from clinical duties during the simulation sessions. To encourage participation, continuing medical education credits were offered to the participants free of charge.

CED demographics such as annual pediatric volume, distance to academic medical center (AMC), and the availability of a pediatric inpatient service were documented. CED's pediatric volume was categorized based on Emergency Medical Services for Children's definitions for pediatric patient visits per year (low $(<1800)$, medium (1800-4999), and 
medium to high (5000-9999)).

A collaborative education team of pediatric intensivists, pediatric emergency physicians, two pediatric critical care transport nurses and a pediatric respiratory therapist from the main AMC in the state of Indiana named "Pediatric Community Outreach Mobile Education PCOME" conducted the study. All members of the educator team had undergone training in simulation and reflective debriefing.

\section{$\underline{\text { Simulations: }}$}

Each CED visit started with a presentation to introduce the educator team, describe the agenda for the day, and provided the participants with the rules and expectations regarding the simulation sessions.

All CED teams participated in a standardized in situ simulation for a 3-month infant patient with a severe AHT presenting to their ED. Simulation sessions took place in the actual CED rooms using each site's actual equipment (e.g. infusion pumps), supplies (e.g. syringes) resources (ex: cognitive aids), and policies/guidelines.

Before each simulation session, participants were oriented to the functionality of the high fidelity simulator (SimNewB ${ }^{\circledR}$ designed by Laerdal, New York, USA). The team was introduced to the embedded participant that was used as a parent. The case was started with a brief scenario that included a patient history and chief complaint. Laboratory data were provided on request on pre-printed laminated cards, including standard point-ofcare testing (e.g., venous blood gas, dextrose, electrolytes, radiologic imaging) (supplementary file 1, 2). 
Each scenario ran for 20 minutes and was followed by a 40-minute post-simulation reflective debriefing. Facilitator-guided post-event debriefing using three phases structure (reaction, analysis, summary) was used to ensure the relevant issues and learning objectives identified during the scenario were addressed. Debriefings were structured to focus on opportunities for improvement resuscitative care of the multi-professional team and to identify knowledge deficits and latent safety threats. We also distributed a pediatric AHT flowchart to each site that incorporates guidelines and recommendations for AHT evaluation and management (Figure. 1) ${ }^{22}$.

\section{Outcome Measures:}

The primary outcome of this study was the quality of stabilization efforts provided to a pediatric patient presenting with AHT as measured by adherence to critical action checklist. Content validity evidence of the checklist was provided through adaptation of established guidelines and a consensus-based approach by a multidisciplinary expert panel of pediatric critical care, pediatric emergency medicine, and pediatric neurosurgery team members. The checklist piloted within our institution prior to use in this study. To further validate the checklist, the checklist was also piloted at four CED sites not included in our study and changes based on learnings from these sites were made as prior to starting this study.

During the simulation sessions, team performance was scored in real-time by two separate facilitators documenting the number of checklist items performed correctly. 
After the simulation was complete, the facilitators discussed differences in their respective scoring to reach a consensus score. This two approach increased the likelihood that if the team was performing two checklist items simultaneously, that one of the facilitators would document item completion. A case performance score was calculated using equal weighting for all subcomponents and dividing by the total number of possible elements to derive the composite adherence score (CAS) on a scale of 0 to 100.

CED providers' self-reported confidence/comfort level managing pediatric patients with abusive head trauma was collected after each simulation session. After each simulation session, participants completed a survey assessing their confidence/comfort regarding the management of AHT in their CED. The participant self-assessment followed a 1 to 5 Likert scale $(1=$ no confidence/understanding and $5=$ complete confidence/ understanding).

\section{Statistical Analysis:}

Basic descriptive statistics were calculated as frequencies (percentages) for categorical variables and medians (inter-quartile ranges) for continuous variables. Associations between hospital characteristics and CAS scores were analyzed using Wilcoxon rank-sum tests for categorical variables and Spearman rank-sum correlation analyses for continuous variables, due to non-linear distribution of CAS scores. Analyses were performed using SAS v9.4 (SAS Institute, Cary, NC).

\section{Results}




\section{Participating Hospitals and Team Characteristics}

Fifty-three multi-professional teams from 18 CED sites located within 100 miles from the main pediatric AMC were enrolled in the project between January 2018 and February 2019. None of the participating CEDs had a separate pediatric ED. Eight of the 18 CEDs are certified adult trauma hospitals: one CED was level 1, two CEDs were level 2, and five CEDs were level 3. A total of 270 participants were involved in the simulations, of which $20.7 \%$ were physicians, $65.2 \%$ were nurses, $9.3 \%$ were respiratory therapists, and $4.8 \%$ were other staff (paramedics, pharmacists, or students). Detailed team characteristics and CED site demographics are shown in Table 1.

\section{Simulation Performance Outcomes}

Out of all tasks, assessment of airway/breathing was the most successfully conducted task by $53 / 53$ teams (100\%) followed by placing an IV/IO access by $52 / 53$ teams $(98 \%)$. Assessment of circulation, neurological exam and full body exposure were conducted by $53 \%, 55 \%$ and $47 \%$ of teams, respectively. Although 43/53 teams (81\%) verbalized the suspicion for AHT and 49/53 teams (94.5\%) verbalized the need for head CT, only 21/53 teams $(39.6 \%)$ used hyperosmolar agent, 4/53 teams (7.5\%) applied cervical spine collar stabilization, and 6/53 teams $(11.3 \%)$ applied neuroprotective measures. The median CAS for all teams was $60.8 \%$.None of the teams were adherent to all AHT checklist stabilization tasks. (Table. 2).

\section{Effects of hospital CEDs characteristics on teams' performance}


No significant difference in the composite adherence score (CAS) was found between CEDs with an inpatient pediatric service and those without onsite pediatric units or CEDs with and without adult trauma center designation. Teams from low to medium pediatric volume CED had significantly higher CAS than teams from medium to high volume CEDs (CAS 67\% vs 53\%, $\mathrm{p}=0.0168$, Table 3). Teams from CED sites closer to the main AMC had higher CAS score compared to CEDs that are further from the AMC $(\mathrm{p}=0.002)$ (Table 3).

\section{Course Evaluation Outcome}

Two hundred sixty-two participants completed post-simulation surveys (8 participants left the session early because of clinical duty). The vast majority of participants (95\%) found the simulation session increased their comfort in the recognition and management of AHT pediatric patients. Ninety-eight percent of participants indicated that they would alter their current practice of AHT management based on the education received during the simulation session (Table 4).

\section{Discussion}

This study demonstrated gaps in the quality of resuscitative care provided to an infant presenting with an abusive head trauma across a diverse spectrum of CEDs in a simulated setting. In this study, our simulation-based assessment revealed variability in the initial recognition of an infant presenting with AHT to CEDs and the quality of resuscitative efforts provided by CED teams. Additionally, it demonstrated differences in quality of 
stabilization efforts among CEDs teams based on their pediatric patient volume or distance from the academic medical center. This is the first study that uses simulationbased methodology to measure the quality of resuscitative care of a pediatric patient with AHT within the actual care setting.

In the US, less than $10 \%$ of injured children are initially cared for at a children's hospital or pediatric trauma center. They more commonly present to the nearest CED for initial work up and stabilization at facilities where pediatric emergency care is a low-frequency event ${ }^{15}$. Outcome disparities between CEDs and pediatric trauma centers for pediatric trauma patients persist, likely secondary to limited resources, distance to appropriate pediatric trauma centers, and a lack of pediatric surgeons and/or specialists available at the $\mathrm{CED}^{23-25}$. Recent studies targeting collaborative improvement programs to enhance the quality of care provided to pediatric trauma patients in CEDs have been successful 26,27 .

Children with AHT are at higher risk for poor outcomes given the younger age, higher incidence of seizure, inconsistent recognition, and lack of pediatric specialists readily available ${ }^{28}$. However, very limited work had done to evaluate and improve CEDs teams providing acute resuscitative care to pediatric patients with AHT through implementing targeted educational interventions ${ }^{29} 30$

Our study revealed several important findings. While $100 \%$ of teams performed initial airway assessment, only half of the teams assessed circulation, neurological status and performed full body exposure. These items represent important steps in the primary 
survey of a pediatric trauma patient ${ }^{31}$. This could be explained by the lack of recognition of a potential trauma given the initial presentation as a "lethargic infant". Hunt et al reported similar deficiencies in the stabilization of children presenting to CEDs in simulated setting making this a potential target for future intervention including team training and providers' competency ${ }^{32}$.

Although $81 \%$ of teams verbalized their concerns regarding a potential AHT during the simulated scenarios, only $7 \%$ of CEDs providers applied an appropriate cervical collar. Similarly, only $11 \%$ of teams elevated the head of bed at 20-30 degrees, and $39 \%$ of teams appropriately used hyperosmolar agent in respond to vital sings changes correlating with increased intracranial pressure. During debriefings, one of the barriers to use cervical collar is the lack of availability of appropriate sizes for an infant patient. It was also noted that most teams failed to apply neuroprotective measures due to the lack of familiarity with the pediatric best practices and the low exposures to these injuries in their CED setting. This could be attributed to the lack of consensus regarding treatment goals in the context of AHT and the difficulty in applying TBI literature guidelines to population with AHT ${ }^{16}$. This is an important finding and clinically relevant since TBI management guidelines in children focuses on preventing secondary brain injury to improve outcomes given that TBI secondary to AHT is a major cause of death and disability and is considered a "silent epidemic" 33,34 .

Despite previous reports that higher pediatric volume CEDs showed better adherence to best practices and established guidelines of care than lower volume CEDs ${ }^{35}$, our study 
showed different findings. This is potentially due to the small sample size and /or to a lack of pediatric trauma-specific guidelines in the CEDs where we conducted our study. Interestingly, in our cohort CEDs closer to the AMC had higher composite adherence scores than more distant CEDs. These finding are consistent with a previous study showing improved quality of resuscitative care in CEDs that are more proximal to the AMC, and this could be explained by the fact that more proximal CEDs have more frequent interaction with the pediatric trauma center and easier access to pediatric specialists and resources ${ }^{36}$.

Our simulation-based initiative was well received by CEDs providers. Ninety-nine of participating providers reported increased comfort in the recognition of AHT. Furthermore, 98\% self-reported increased comfort in management of a pediatric patient presenting with AHT to their ED. This is likely due to the immediate post simulation debriefing conducted after each scenario that allowed all team members to reflect on their own performance and provided opportunities to implement suggestions for continuing learning to improve future individual and team performance. Samples of the participants' reflective comments were "Very informative without making you feel bad for not knowing all the answers. We need more of these", "No one was intimidating but gave great constructive criticism" Additionally, the AHT flowcharts we distributed during the debriefings provided a cognitive gain among providers (figure 1).

Our study has several limitations. We measured the quality of resuscitative care in simulated setting using a checklist that has not been validated using a known validation 
framework. Despite a growing body of literature on AHT as a distinct form of TBI, there remains a dearth of evidence AHT acute targeted therapies making it difficult to extrapolate accidental TBI guidelines to AHT population. To mitigate that, relevant items in the checklist were derived from best practices and guidelines and were reviewed by a multidisciplinary expert group of critical care physicians, pediatric emergency physicians, a pediatric trauma surgeon and a pediatric neurosurgeon at our institution. It was also pilot tested prior to its implementation in the study. Study results may be limited by the relatively close geography of the participant sites to the AMC, and the results may not be generalizable to CEDs that are further from the AMC. Finally, it is difficult to ensure that simulated scenarios are sufficiently realistic, and it is challenging to demonstrate that learning from simulation sessions will translate into improved care in patient care.

\section{Conclusions}

Using in situ, high fidelity simulation our study revealed high variability in quality of resuscitative care provided to children with abusive head trauma across a diverse set of community emergency departments. We identified several deficiencies in the initial stabilization of children presenting with AHT that serve as a basis for the development of future educational interventions targeted at providers in CEDs. Future research should investigate whether interventions focused on these deficiencies can improve the quality of care provided in simulated and potentially clinical setting, and ultimately, improve the outcomes of children with AHT presenting to CEDs. 


\section{Reference}

1. Child Maltreatment https://www.acf.hhs.gov/cb/resource/childmaltreatment-2017. Published 2017. Accessed 02/24/, 2020.

2. Jenny C, Hymel KP, Ritzen A, et al. Analysis of missed cases of abusive head trauma. JAMA. 1999;281(7):621-626.

3. Boop S, Axente M, Weatherford B, et al. Abusive head trauma: an epidemiological and cost analysis. J Neurosurg Pediatr. 2016;18(5):542-549.

4. DiScala C, Sege R, Li G, Reece RM. Child abuse and unintentional injuries: a 10-year retrospective. Arch Pediatr Adolesc Med. 2000;154(1):16-22. 
5. Altman RL, Canter J, Patrick PA, et al. Parent education by maternity nurses and prevention of abusive head trauma. Pediatrics. 2011;128(5):e1164-1172.

6. Amanullah S, Schlichting LE, Linakis SW, et al. Emergency Department Visits Owing to Intentional and Unintentional Traumatic Brain Injury among Infants in the United States: A Population-Based Assessment. J Pediatr. 2018;203:259-265 e251.

7. Duhaime AC, Alario AJ, Lewander WJ, et al. Head injury in very young children: mechanisms, injury types, and ophthalmologic findings in 100 hospitalized patients younger than 2 years of age. Pediatrics. 1992;90(2 Pt 1):179-185.

8. Keshavarz R, Kawashima R, Low C. Child abuse and neglect presentations to a pediatric emergency department. J Emerg Med. 2002;23(4):341-345.

9. Trokel M, Waddimba A, Griffith J, Sege R. Variation in the diagnosis of child abuse in severely injured infants. Pediatrics. 2006;117(3):722-728.

10. Whitfill T, Auerbach M, Scherzer DJ, et al. Emergency Care for Children in the United States: Epidemiology and Trends Over Time. J Emerg Med. 2018;55(3):423-434.

11. Tiyyagura G, Gawel M, Koziel JR, et al. Barriers and Facilitators to Detecting Child Abuse and Neglect in General Emergency Departments. Ann Emerg Med. 2015;66(5):447-454.

12. Auerbach M, Whitfill T, Gawel M, et al. Differences in the Quality of Pediatric Resuscitative Care Across a Spectrum of Emergency Departments. JAMA Pediatr. 2016;170(10):987-994. 
13. Gausche-Hill M, Ely M, Schmuhl P, et al. A national assessment of pediatric readiness of emergency departments. JAMA Pediatr. 2015;169(6):527-534.

14. Hudgins JD, Monuteaux MC, Bourgeois FT, et al. Complexity and Severity of Pediatric Patients Treated at United States Emergency Departments. J Pediatr. 2017;186:145-149 e141.

15. Hansen M, Fleischman R, Meckler G, et al. The association between hospital type and mortality among critically ill children in US EDs. Resuscitation. 2013;84(4):488-491.

16. Iqbal O'Meara AM, Sequeira J, Miller Ferguson N. Advances and Future Directions of Diagnosis and Management of Pediatric Abusive Head Trauma: A Review of the Literature. Front Neurol. 2020;11:118.

17. Nishisaki A, Keren R, Nadkarni V. Does simulation improve patient safety? Self-efficacy, competence, operational performance, and patient safety. Anesthesiol Clin. 2007;25(2):225-236.

18. Brydges R, Hatala R, Zendejas B, et al. Linking simulation-based educational assessments and patient-related outcomes: a systematic review and metaanalysis. Acad Med. 2015;90(2):246-256.

19. Cheng A, Auerbach M, Hunt EA, et al. Designing and conducting simulationbased research. Pediatrics. 2014;133(6):1091-1101.

20. Patterson MD, Geis GL, Falcone RA, et al. In situ simulation: detection of safety threats and teamwork training in a high risk emergency department. BMJ Qual Saf. 2013;22(6):468-477. 
21. Geis GL, Pio B, Pendergrass TL, et al. Simulation to assess the safety of new healthcare teams and new facilities. Simul Healthc. 2011;6(3):125-133.

22. Schouten MC, van Stel HF, Verheij TJ, et al. A screening protocol for child abuse at out-of-hours primary care locations: a descriptive study. BMC Fam Pract. 2016;17(1):155.

23. Petrosyan M, Guner YS, Emami CN, et al. Disparities in the delivery of pediatric trauma care. J Trauma. 2009;67(2 Suppl):S114-119.

24. Jarman MP, Castillo RC, Carlini AR, et al. Rural risk: Geographic disparities in trauma mortality. Surgery. 2016;160(6):1551-1559.

25. Densmore JC, Lim HJ, Oldham KT, et al. Outcomes and delivery of care in pediatric injury. J Pediatr Surg. 2006;41(1):92-98; discussion 92-98.

26. Kelley-Quon LI, Crowley MA, Applebaum H, et al. Academic-community partnerships improve outcomes in pediatric trauma care.J Pediatr Surg. 2015;50(6):1032-1036.

27. Hunt EA, Heine M, Hohenhaus SM, et al. Simulated pediatric trauma team management: assessment of an educational intervention. Pediatr Emerg Care. 2007;23(11):796-804.

28. Duhaime AC, Durham S. Traumatic brain injury in infants: the phenomenon of subdural hemorrhage with hemispheric hypodensity ("Big Black Brain"). Prog Brain Res. 2007;161:293-302.

29. Alphonso A, Auerbach M, Bechtel K, et al. Development of a Child Abuse Checklist to Evaluate Prehospital Provider Performance. Prehosp Emerg Care. 2017;21(2):222-232. 
30. Tiyyagura G, Asnes AG, Leventhal JM, et al. Impact of Project ECHO on Community ED Providers' Perceptions of Child Abuse Knowledge and Access to Subspecialists for Child Abuse and Neglect. Acad Pediatr. 2019;19(8):985987.

31. Duff JP, Topjian A, Berg MD, et al. 2018 American Heart Association Focused Update on Pediatric Advanced Life Support: An Update to the American Heart Association Guidelines for Cardiopulmonary Resuscitation and Emergency Cardiovascular Care. Circulation. 2018;138(23):e731-e739.

32. Hunt EA, Hohenhaus SM, Luo X, et al. Simulation of pediatric trauma stabilization in 35 North Carolina emergency departments: identification of targets for performance improvement. Pediatrics. 2006;117(3):641-648.

33. Agrawal S, Branco RG. Neuroprotective measures in children with traumatic brain injury. World J Crit Care Med. 2016;5(1):36-46.

34. Langlois JA, Marr A, Mitchko J, et al. Tracking the silent epidemic and educating the public: $C D C$ 's traumatic brain injury-associated activities under the TBI Act of 1996 and the Children's Health Act of 2000. J Head Trauma Rehabil. 2005;20(3):196-204.

35. Kessler DO, Walsh B, Whitfill T, et al. Disparities in Adherence to Pediatric Sepsis Guidelines across a Spectrum of Emergency Departments: A Multicenter, Cross-Sectional Observational In Situ Simulation Study. J Emerg Med. 2016;50(3):403-415 e401-403.

36. Abulebda K, Whitfill T, Montgomery EE, et al. Improving Pediatric Diabetic Ketoacidosis Management in Community Emergency Departments Using a 
Simulation-Based Collaborative Improvement Program. Pediatr Emerg Care. 2019. 


\section{Tables}

Table 1. Characteristics of Participating Teams and Community Emergency Departments

\begin{tabular}{|l|l|}
\hline Team characteristics & \\
\hline Number of teams & 53 \\
\hline Number of Participants & 270 \\
\hline Number of MDs, number (\%) & $56(20.7 \%)$ \\
\hline Number of RNs, number (\%) & $176(65.2 \%)$ \\
\hline Number of RTs, number (\%) & $25(9.3 \%)$ \\
\hline Number of Others, number (\%) & $13(4.8 \%)$ \\
\hline CED characteristics & \\
\hline Number of CEDs & 18 \\
\hline CED pediatric volume & \\
\hline Low & $2(11.2 \%)$ \\
\hline Medium & $8(44.4 \%)$ \\
\hline Medium to high & $8(44.4 \%)$ \\
\hline Affiliation with AMC & $5(27.8 \%)$ \\
\hline Distance from the AMC (miles) & $46(19,60)$ \\
\hline Presence of inpatient pediatric unit & $166.7 \%)$ \\
\hline
\end{tabular}




\begin{tabular}{|l|l|}
\hline Certified Adult Trauma Hospitals & 8 (44\%) \\
\hline Data presented as number (\%) or median (25th, 75th \\
interquartile range) \\
MD: Physician; RN: nurse; RT: respiratory therapist; CED: \\
community emergency department; AMC: academic medical \\
center; ICU: intensive care unit.
\end{tabular}

Table 2. Abusive Head Trauma Management Adherence Checklist.

\begin{tabular}{|l|l|l|}
\hline$\#$ & Item & $\mathrm{N}(\%)$ \\
\hline 1 & $\begin{array}{l}\text { Assesses airway/breathing } \\
\text { In first 5 min }\end{array}$ & $53(100 \%)$ \\
\hline 2 & $\begin{array}{l}\text { Assesses circulation } \\
\text { In first 5 min }\end{array}$ & $28(52.8 \%)$ \\
\hline 3 & $\begin{array}{l}\text { Assesses neurological status } \\
\text { In first 5 min }\end{array}$ & $29(54.7 \%)$ \\
\hline 4 & Performs full body exposure & $25(47.2 \%)$ \\
\hline 5 & Places IV/IO & $52(98.1 \%)$ \\
\hline 6 & $\begin{array}{l}\text { Orders relevant labs (basic metabolic panel } \\
\text { or point-of-care blood gas) }\end{array}$ & $40(75.5 \%)$ \\
\hline 7 & Verbalizes concerns for AHT & $43(81.1 \%)$ \\
\hline 8 & Verbalizes concerns of increased ICP & $35(66.0 \%)$ \\
\hline
\end{tabular}




\begin{tabular}{|c|c|c|}
\hline 9 & Applies C-collar stabilization & $4(7.5 \%)$ \\
\hline 10 & Verbalizes the need of head CT & $49(92.5 \%)$ \\
\hline 11 & $\begin{array}{l}\text { Applies neuroprotective measures: } \\
\text { HOB elevated 20-30 degrees }\end{array}$ & $6(11.3 \%)$ \\
\hline 12 & $\begin{array}{l}\text { Uses appropriate hyperosmolar agent: } \\
\text { 3\% HTS: } 5-10 \mathrm{ml} / \mathrm{kg} \\
\text { Mannitol: } 0.25-1 \mathrm{gm} / \mathrm{kg}\end{array}$ & $21(39.6 \%)$ \\
\hline 13 & $\begin{array}{l}\text { Considers patient intubated prior to } \\
\text { transport }\end{array}$ & $35(66 \%)$ \\
\hline 14 & $\begin{array}{l}\text { Notifies child protective service/Social } \\
\text { worker }\end{array}$ & $32(60.4 \%)$ \\
\hline 15 & Arranges for tertiary center transport & $31(58.5 \%)$ \\
\hline & Complete Adherence Score (CAS) & $60.8 \%$ \\
\hline
\end{tabular}


Table 3. Effects of Hospital Characteristics on Composite Adherence Scores

\begin{tabular}{|c|c|c|}
\hline Variable & CAS & $p$-value \\
\hline $\begin{array}{l}\text { Availability of inpatient pediatric services } \\
\text { - Yes } \\
\text { - No }\end{array}$ & $\begin{array}{l}0.60(0.47-0.73) \\
0.63(0.60-0.73)\end{array}$ & .2361 \\
\hline Distance from the AMC & -0.4129 & .0021 \\
\hline $\begin{array}{l}\text { Affiliation with the AMC } \\
\therefore \text { Yes } \\
\circ \text { No }\end{array}$ & $\begin{array}{l}0.53(0.40-0.67) \\
0.63(0.53-0.73)\end{array}$ & .0662 \\
\hline $\begin{array}{l}\text { CED pediatric patient volume } \\
\text { - Low and medium } \\
\text { - Medium to high }\end{array}$ & $\begin{array}{l}0.67(0.60-0.73) \\
0.53(0.40-0.67)\end{array}$ & .0168 \\
\hline $\begin{array}{l}\text { Designated adult trauma center } \\
\circ \text { Yes } \\
\circ \text { No }\end{array}$ & $\begin{array}{l}0.53(0.53-0.63) \\
0.60(0.53-0.73)\end{array}$ & .5295 \\
\hline $\begin{array}{l}\text { Data presented as median }(25 \text { th, } 75 \text { th interc } \\
\text { values, for categorical variables and Spearn } \\
\text { continuous variables. }\end{array}$ & $\begin{array}{l}\text { artile range), with } \\
\text { an correlation coeff }\end{array}$ & $\begin{array}{l}\text { Vilcoxon } \mathrm{p} \\
\text { cients for }\end{array}$ \\
\hline
\end{tabular}


CAS: composite adherence score; AMC: academic medical center

Table 4: Participant's Evaluation of the Simulation Session

\begin{tabular}{|c|c|c|c|c|c|}
\hline & $\begin{array}{l}\text { Strongly } \\
\text { disagree }\end{array}$ & Disagree & Neutral & Agree & $\begin{array}{l}\text { Strongly } \\
\text { agree }\end{array}$ \\
\hline $\begin{array}{l}\text { 1. I am more comfortable } \\
\text { with identifying AHT and } \\
\text { associated symptoms }\end{array}$ & 0 & 0 & $\begin{array}{l}2 \\
(1 \%)\end{array}$ & $\begin{array}{l}106 \\
(40 \%)\end{array}$ & $\begin{array}{l}154 \\
(59 \%)\end{array}$ \\
\hline $\begin{array}{l}\text { 2. I am more comfortable } \\
\text { with the management of an } \\
\text { AHT patient }\end{array}$ & 0 & 0 & $\begin{array}{l}4 \\
(2 \%)\end{array}$ & $\begin{array}{l}111 \\
(42 \%)\end{array}$ & $\begin{array}{l}147 \\
(56 \%)\end{array}$ \\
\hline $\begin{array}{l}\text { 3. I'm likely to alter my } \\
\text { current management of a } \\
\text { pediatric patient with AHT }\end{array}$ & 0 & 0 & $\begin{array}{l}4 \\
(2 \%)\end{array}$ & $\begin{array}{l}116 \\
(44 \%)\end{array}$ & $\begin{array}{l}142 \\
(54 \%)\end{array}$ \\
\hline \multicolumn{6}{|l|}{$\begin{array}{l}\text { Surveys filled by } 262 \text { participants } \\
\text { AHT: abusive head trauma }\end{array}$} \\
\hline
\end{tabular}




\section{Figure}

Figure 1: Abusive Head Trauma Assessment and Management Flowsheet 
Injury compatible with history and corresponding with age of child?

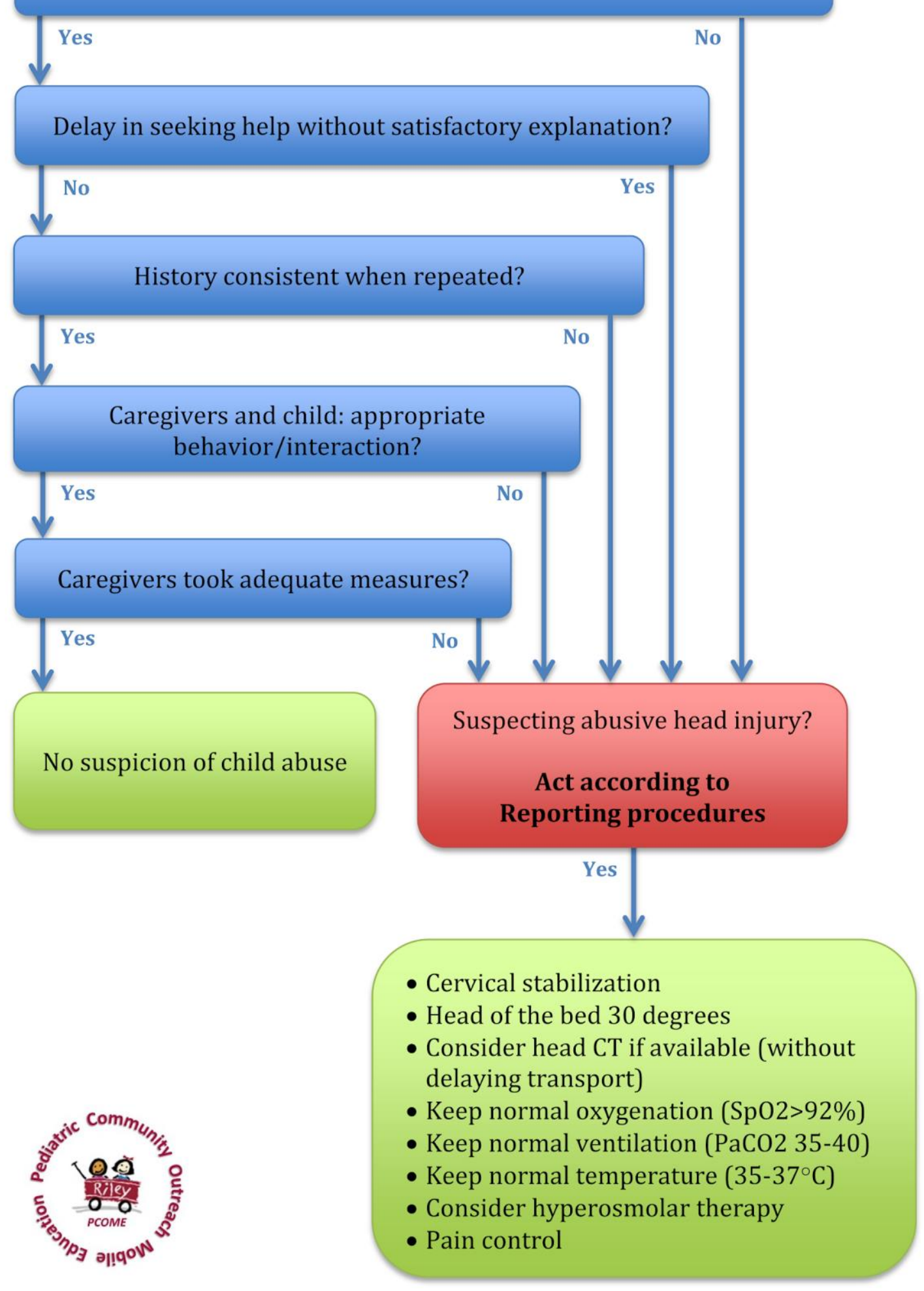


Modified with permission from Schouten et al, A screening protocol for child abuse at out-of-hours primary care locations: a descriptive study, BMC Fam Pract. 2016 Nov 8;17(1):155. 